\title{
Estabilidad de color de tres materiales dentales provisorios sumergidos en dos agentes pigmentantes
}

\author{
Color stability of three provisional dental materials submerged in two \\ pigmenting agents
}

Samantha Chuquiano Bringas (b) ${ }^{1 a}$, Karen Arroyo Rivera (b) ${ }^{1 a, b}$, Rafael Morales Vadillo $1 a, b, c$

\section{RESUMEN}

Objetivo: Determinar la estabilidad de color de tres materiales dentales provisorios al ser sumergidos en dos agentes pigmentantes. Materiales y métodos: es un estudio experimental, analítico, prospectivo y longitudinal. Se elaboraron un total de 270 discos de $15 \mathrm{~mm}$ de diámetro y $1 \mathrm{~mm}$ de espesor, con 3 materiales dentales provisorios: resina bisacrílica (Luxatemp Star) color A1, resina acrílica (Alike) color 59 y PMMA de autocurado (Vitalloy) color 59 siendo sumergidos 30 de cada muestra en chicha morada, café y agua destilada (control). Las lecturas de color se realizaron de manera inicial, a los 7 y 28 días usando el espectofotómetro VITA $®$ EasyShade $₫$ V con el sistema CIELab. Resultados: La resina acrílica presentó mayor estabilidad de color comparada con la resina bisacrílica y PMMA de autocurado $(p<0.01)$.El café presentó el mayor cambio de color $(p<0,001)$. Después de 28 días de almacenamiento, hubo cambio de color en todos los materiales analizados. Conclusión: La resina acrílica presentó la mayor estabilidad de color. El café causó el mayor cambio de color y el tiempo de inmersión fue determinante para la estabilidad de color en los materiales analizados.

Palabras claves: Color; Material temporal;-Restauración dental provisional; Resina-acrílica; Polimetil Metacrilato; Café. (Fuente: DeCS BIREME)

\section{ABSTRACT}

Objective: To determine the color stability of three provisional dental materials when immersed in two pigment agents. Materials and methods: it is an experimental, analytical, prospective and longitudinal study. A total of 270 discs $15 \mathrm{~mm}$ in diameter and $1 \mathrm{~mm}$ thick were made with 3 temporary dental materials: bisacrylic resin (Luxatemp Star) color A1, acrylic resin (Alike) color 59 and PMMA self-curing (Vitalloy) color 59 being submerged 30 of each sample in purple corn, coffee and distilled water (control). Color readings are used initially, at 7 and 28 days using the VITA ${ }^{\circledR}$ EasyShade ${ }^{\circledR} \vee$ spectrophotometer with the CIELab system. Results: The acrylic resin presented greater color stability compared to the bisacrylic resin and PMMA selfcuring $(p<0.01)$. The coffee presented the greatest color change $(p<0.001)$. After 28 days of storage, there was a color change in all the materials analyzed. Conclusion: Acrylic resin has the highest color stability. The coffee caused the greatest color change and the immersion's time was decisive for the color stability.

Keywords: Color; Temporary material; Acrylic resins; Polymethyl methacrylate; Coffee. (Source: MeSH NLM)

\footnotetext{
${ }^{1}$ Facultad de Odontología, Universidad de San Martín de Porres. Lima, Perú

${ }^{a}$ Cirujano dentista

${ }^{b}$ Especialista

${ }^{\mathrm{C}}$ Doctor
}

Este es un artículo de acceso abierto distribuido bajo la licencia Creative Commons Atribución 4.0 Internacional (CC BY 4.0) https://creativecommons.org/licenses/by/4.0/deed.es

Correspondencia:

Samantha Chuquiano

Correo electrónico: sam_ysabel14@hotmail.com

Citar como: Chuquiano Bringas S, Arroyo Rivera K, Morales Vadillo R. Estabilidad de color de tres materiales dentales provisorios sumergidos en dos agentes pigmentantes. KIRU. 2021;18(1):11-18. https://doi.org/10.24265/kiru.2021.v18n1.02 


\section{INTRODUCCIÓN}

La prótesis provisional es la que se establece por un período de tiempo mientras que se realiza la restauración o prótesis definitiva ${ }^{(1,2)}$.

En los tratamientos de rehabilitación oral en el sector anterior es importante cuidar la estética y naturalidad de los dientes; por ese motivo, se tiene que tener un material en función de la estabilidad de color ${ }^{(3-6)}$.

Es importante seleccionar un material restaurador con mayor resistencia a diferentes pigmentaciones y líquidos a los que están expuestos, con el fin de optimizar la estética de las restauraciones realizadas ${ }^{(3,7,8)}$. La literatura indica que el polimetilmetacrilato (PMMA) es el material de elección cuando las restauraciones provisionales se confeccionan utilizando técnicas indirectas. Si bien la resina acrílica se viene utilizando desde hace años, el mercado ofrece otro tipo de material llamada resina bisacrílica, basada en un sistema de pasta-pasta (2). También tienen buenas propiedades mecánicas y una técnica muy sencilla de confección de los provisorios $^{(9)}$.

La estabilidad de color es el grado de equilibrio de un color dado por un colorímetro que a la acción de un agente pigmentante cambia en el transcurso del tiempo ${ }^{(10)}$.

En el campo de la investigación de los materiales odontológicos, el espectrofotómetro es un instrumento ampliamente usado, que mide el color usando el Sistema de la Comisión Internacional de L'E clairage (CIE Lab), el cual tiene tres coordenadas espaciales: $L$, $a$ y $b$. $L$ se refiere a la luminosidad del material, a la cantidad de color rojo-verde y $b$ de amarillo-azul. Para cuantificar el color numéricamente se utiliza el valor $\Delta E$, que se obtiene a través de la siguiente fórmula. El resultado nos dará el grado de alteración del color, por lo que a mayor cercanía de 0 habrá mayor estabilidad de color $(11-20)$

$$
\Delta \mathrm{E}=\left[\left(\mathrm{L}_{\mathrm{l}}-\mathrm{L}_{0}\right)^{2}+\left(\mathrm{a}_{1}-\mathrm{a}_{0}\right)^{2}+\left(\mathrm{b}_{1}-\mathrm{b}_{0}\right)^{2}\right]^{1 / 2}
$$

Con las diferentes gamas de materiales dentales provisorios que tenemos en el mercado, nos pone en duda cuál es el ideal para nuestros pacientes al colocárselos en boca, como la seguridad y certeza de que no llegue a variar su coloración y así mantenga su estabilidad con los residuos de alimentos que pueda haber en boca.
En el estudio se determinó la estabilidad de color de tres materiales dentales provisorios al ser sumergidos en agua destilada, café y chicha morada a los 7 y 28 días respectivamente. Así mismo, comparar los agentes pigmentantes a los 7 y 28 días de cada grupo; y contrastar la estabilidad de color de tres materiales dentales provisorios sumergidos en café, chicha morada y agua destilada (control) a los 7 y 28 días.

\section{MATERIAL Y MÉTODOS}

El estudio es experimental, analítico, prospectivo y longitudinal. La muestra estuvo conformada por 270 discos de acrílico, divididos en 90 muestras de cada tipo de material dental provisorio, confeccionadas según el ISO $4049^{(21)}$. El muestreo fue no probabilístico por conveniencia.

Según los parámetros obtenidos en la prueba piloto se obtuvo un tamaño mínimo de dos muestras por grupo. Sin embargo se amplió la muestra a 30 en cada grupo para compensar las posibles pérdidas de cuerpos de prueba durante el proceso de laboratorio. Fueron incluidos en el estudio Materiales restauradores provisionales y discos de acrílico de $15 \mathrm{~mm}$ diámetro y $1 \mathrm{~mm}$ de espesor. Se excluyeron las muestras que no presentaran las dimensiones determinadas.

La dosificación de las resinas acrílicas y el tiempo de polimerización fue de acuerdo al fabricante, así para la resina acrílica y el PMMA de autocurado se mezcló 300 gr. de polvo con $100 \mathrm{ml}$ de líquido (proporción 3:1), la resina bisacrílica se dispensó con puntas de automezcla que vienen ya proporcionadas (proporción 10:1). Las 270 muestras se realizaron del negativo de un molde metálico de $15 \mathrm{~mm}$ de diámetro por $1 \mathrm{~mm}$ de grosor que estaba sobre una matriz circular de silicona de adición realizado en el laboratorio dental de la Facultad de Odontología de la Universidad de San Martín de Porres, con el fin que las muestras sean estandarizadas según la disposición ISO $4049^{(21)}$ y especificación No.27 del $\operatorname{ADA}^{(6,9,22)}$.

Para ello, se colocó el molde sobre una platina de vidrio previamente aislada y se vació el contenido de la mezcla. Una vez polimerizado se retiró del molde y fueron pulidos tanto los bordes como superficie de las muestras siguiendo las indicaciones de cada fabricante, usando fresas de grano medio, fino y pelo de marta con un micromotor dental (NSK,Japón).

\section{-Exposición a los agentes pigmentantes:}


Se escogió 90 muestras de cada tipo de material dental provisorio para ser sumergido en 2 diferentes agentes pigmentantes más el control, teniendo: 30 muestras de cada material sumergidas en café, chicha morada y agua destilada respectivamente.

La preparación del café fue 6grs. de café (Nescafé Kirma Original) en $500 \mathrm{ml}$. de agua destilada. La preparación de la chicha morada fue $1 / 2 \mathrm{~kg}$. de maíz morado en una olla con $1000 \mathrm{ml}$ de agua destilada, se esperó que hierva para tener la solución. Cada muestra fue sumergida en su propio recipiente con $6 \mathrm{ml}$. de solución respectivamente a temperatura ambiente.
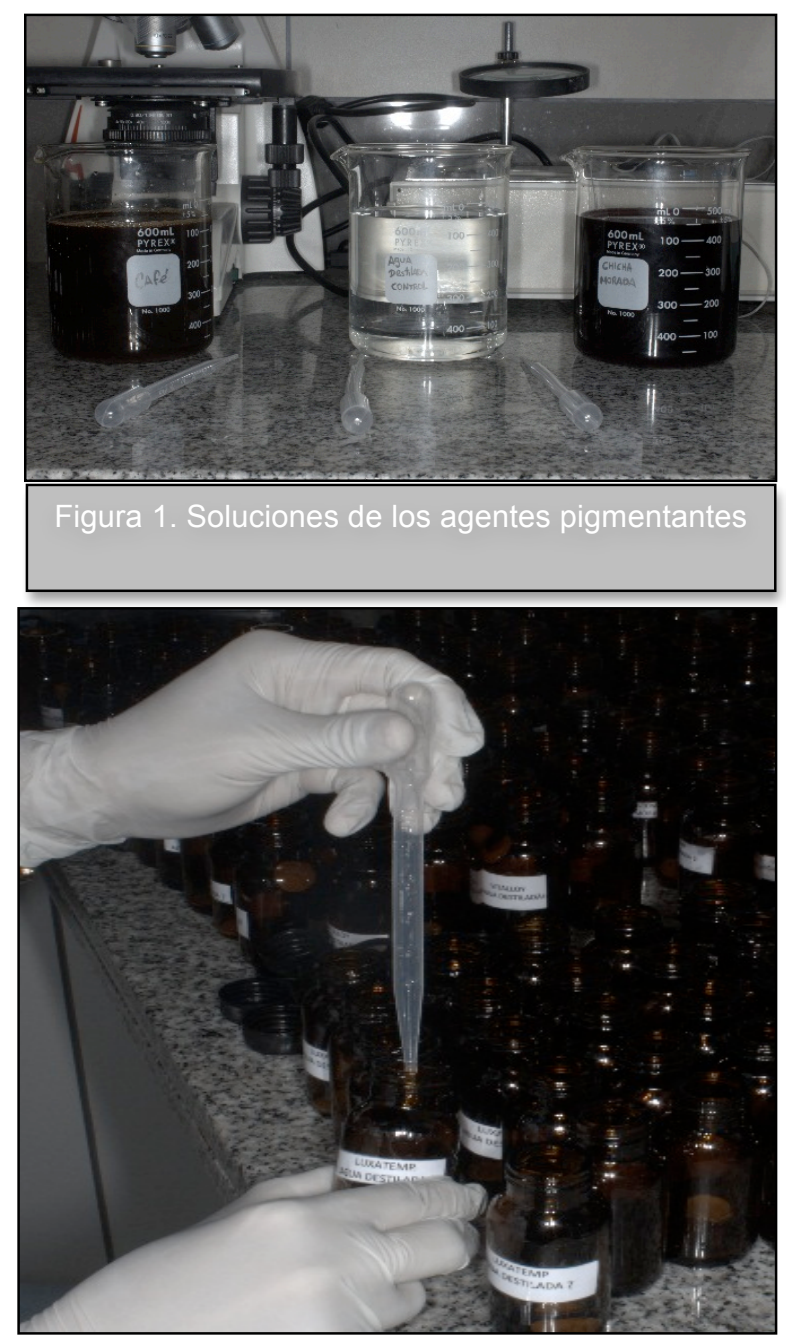

Figura 2. Colocación de los agentes pigmentantes en los envases.

\section{Análisis de la estabilidad de color:}

El color se registró de manera inicial antes de la inmersión en los agentes colorantes dejando todas las muestras 1 día previo en agua destilada que cumple con el grado 2 de (ISO $3696: 2004)^{23}$ a temperatura ambiente $37^{\circ} \mathrm{C}$ (ISO 11405:2003) ${ }^{24}$. Posteriormente se colocó en los agentes pigmentantes respectivos siendo cambiados cada 2 días a temperatura ambiente $37^{\circ} \mathrm{C}$ en la estufa de laboratorio ECOCell 55 (MMM, Alemania).

Nuevas lecturas de color se realizaron a los 7 y 28 días de la exposición de los agentes pigmentantes utilizando el Espectofotómetro VITA $₫$ EasyShade $®$ V. La hora promedio para cada sesión de toma de color fue 9:00 am 11:00 am. Además, para la calibración del equipo se usó un fondo blanco cada 10 disparos con el fin que no se alteren las lecturas.

La recolección de datos se realizó con una Ficha de recolección para cada muestra respectiva en el programa Microsoft Excel 2013. Se evaluó la normalidad con la prueba de Kolmogorov-Smirnov. Para la comparación entre los 7 y 28 días de cada grupo se usó la prueba de los rangos con signo de Wilcoxon cuando por lo menos uno de los grupos no se asemejaba a la distribución normal. Por el contrario, la prueba $t$ de Student para grupos relacionados.

Las comparaciones por soluciones pigmentantes entre diferentes materiales se realizó con la prueba Kruskal Wallis con comparaciones múltiples de Dunn. Así mismo, cuando se utilizó la prueba de ANOVA se realizaron las comparaciones múltiples con el test HSD de Tukey.

Para el análisis se usó el programa Statistical Package for Social Sciences (SPSS, Chicago, IL, USA) versión 23.0 en español y el programa GraphPad Prism versión 5.01 para Windows (Graph Pad Software Inc., La Jolla, CA, USA). Todas las pruebas estadísticas se evaluaron con un nivel de significancia $\mathrm{P}<0.05$.

Consideraciones éticas. Esta investigación conto con la aprobación del Comité de ética de la Facultad de Odontología dela Universidad de San Martin de Porres, Perú. 


\section{RESULTADOS}

Tabla 1. Comparación de estabilidad de color de tres materiales dentales provisorios sumergidos en Café, Chicha Morada y Agua destilada (control) a los 7 días y 28 días

\section{Estabilidad de color con agua destilada}

\begin{tabular}{llcc}
\hline Material provisional & $\mathbf{7}$ días & $\mathbf{2 8}$ días & Sign. \\
\hline Vitalloy & 9,58 & 9,34 & 0,644 \\
Alike & 4,74 & 6,41 & 0,003 \\
Luxatemp Star & 5,33 & 5,01 & 0,894 \\
\hline
\end{tabular}

\section{Estabilidad de color con café}

\begin{tabular}{llcc}
\hline Material provisional & $\mathbf{7}$ días & $\mathbf{2 8}$ días & Sign. \\
\hline Vitalloy & 11,30 & 22,25 & 0,000 \\
Alike & 8,22 & 10,54 & 0,010 \\
Luxatemp Star & 21,66 & 30,66 & 0,000
\end{tabular}

Estabilidad de color con chicha morada

\begin{tabular}{llll}
\hline Material provisional & $\mathbf{7}$ días & $\mathbf{2 8}$ días & Sign. \\
\hline Vitalloy & 8,87 & 15,34 & 0,000 \\
Alike & 5,88 & 8,78 & 0,000 \\
Luxatemp Star & 12,38 & 20,75 & 0,000
\end{tabular}

Prueba de Wilcoxon Sign. $=(p<0,05)$.

A los 7 días sumergidos en cada agente colorante más el control se encontró mejor estabilidad de color en la resina acrílica Alike. Sin embargo, a los 28 días en el agua destilada la resina bisacrílica tuvo mejor estabilidad de color a comparación de la resina acrílica y PMMA de autocurado. Con respecto a los 28 días de exposición de las muestras en café y chicha morada la resina acrílica tuvo mejor estabilidad de color que las demás.
La estabilidad de color de cada material varia y aumenta según el periodo de tiempo en los diversos agentes pigmentantes.

Sin embargo, en el agua destilada tanto en el acrílico de autocurado como la resina bisacrílica pasado los 28 días no existe cambio de color significativo en relación a lo observado a los 7 días. 


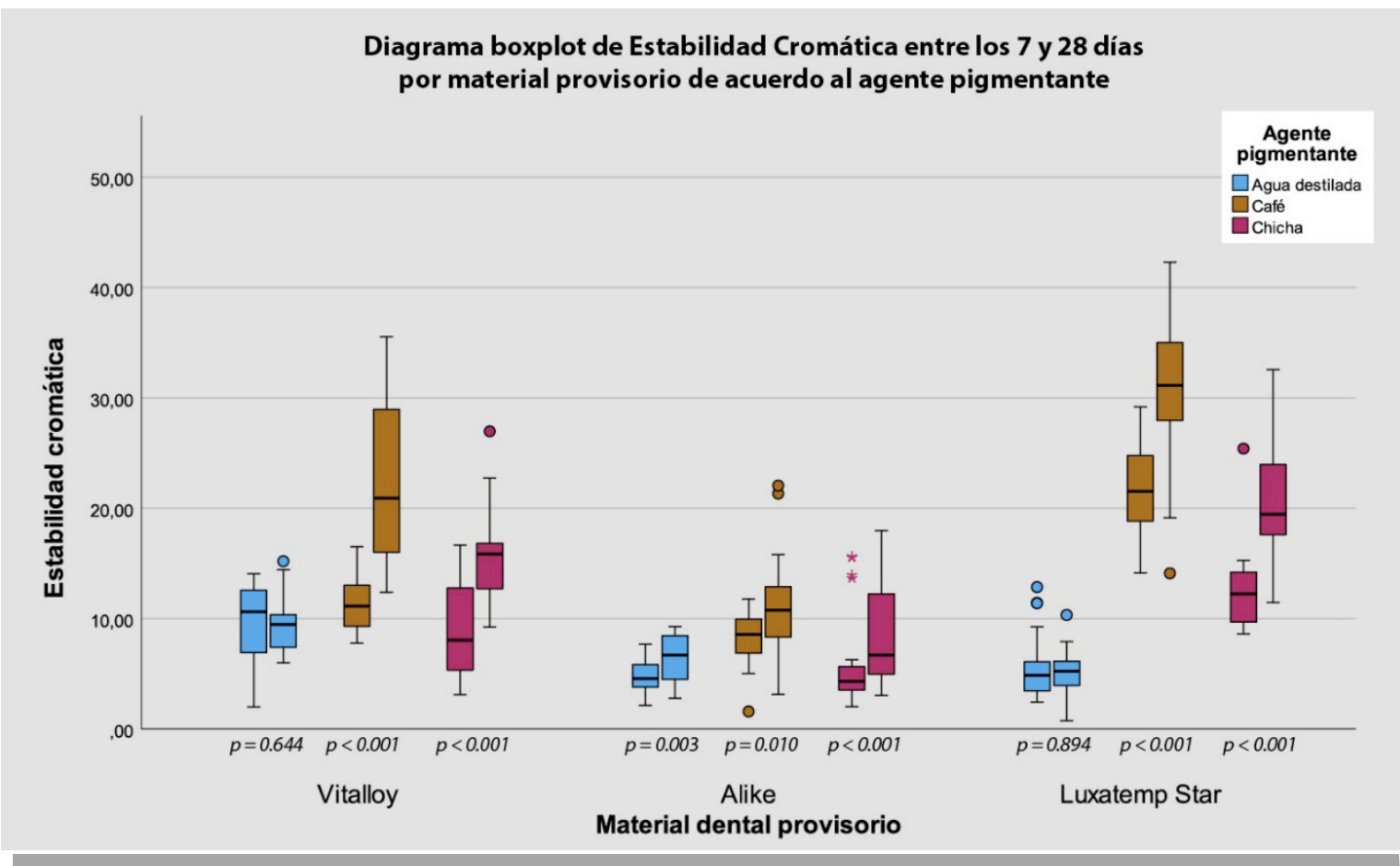

Figura 3. Boxplot de la estabilidad de color entre los 7 y 28 días por material

En un análisis comparativo de la estabilidad de color entre resina acrílica y PMMA de autocurado, se observó una diferencia estadísticamente significativa y favorable para la resina acrílica $(p<0,001)$.

Posteriormente, al analizar específicamente la resina bisacrílica probada, se observó una diferencia estadísticamente significativa en el análisis comparativo entre todos los materiales, que muestra valores de estabilidad de color más altos para la resina acrílica Alike ( $p$ $<0,001)$.

\section{DISCUSIÓN}

Conforme al estudio en el grupo control de agua destilada hubo una diferencia significativa a los 7 días entre la resina acrílica con el PMMA autocurado y la resina bisacrílica. En el estudio Rutkunas $V$ et al. (2009) ${ }^{(17)}$ refiere que luego de repulir las muestras control sumergidas en agua destilada a las $24 \mathrm{hrs}$ el PMMA de autocurado no presentó cambio de color como la resina bisacrílica. Costa í et al. (2018) $^{(18)}$ menciona que la resina acrílica presenta mayor estabilidad de color que resina bisacrílica a los 7 días de estar sumergido en agua destilada. Sin embargo, Rutkunas $\vee$ et al.
(2010) ${ }^{(25)}$ a los 7 días la resina bisacrílica fue el que tuvo mejor estabilidad de color en agua destilada comparado a otras resinas bisacrílicas y acrílicas.

Al cabo de 28 días hubo un cambio siendo la resina bisacrílica quien tuviera mejor estabilidad de color. En el estudio de Sham AS et al. (2004) ${ }^{{ }^{(5)}}$ señala que en 20 días las muestras de resina acrílica al ser sumergidas en agua destilada tienen menor estabilidad de color que la resina bisacrílica.

Al ser las muestras sumergidas en café tanto a los 7 y 28 días se encontró que la resina acrílica tiene la mayor estabilidad de color con respecto al PMMA de autocuraso y resina bisacrílica. Mazaro J. et al. (2015) ${ }^{(7)}$ menciona que la resina acrílica tuvo mayor estabilidad de color que las resinas bisacrílicas siendo la resina bisacrílica la más inestable tanto a los 7 dias como 15 días de inmersión. Así mismo, Torres Loaiza, D. C. et al. (2018) ${ }^{(19)}$ lo refiere en un periodo de 24 horas, teniendo como resultado que la resina acrílica tuvo menor cambio de color a diferencia de la resina bisacrílica. Blasi A. et al. (2011) ${ }^{(3)}$ menciona que las muestras usadas (resina bisacrílica y resina acrílica) tuvieron un termociclado equivalente a 1 mes de uso del material en 
boca. Ambos materiales usados cambiaron de color pero el material que sufrió menor cambio de color fue la resina acrílica. La sustancia que tuvo mayor pigmentación en las muestras fue el café. De acuerdo con Mickeviciute $\mathrm{E}$, et al. (2016) $^{(1)}$, el mayor cambio de color fue con el agente pigmentante café en comparación con la Coca-Cola.

Respecto a los resultados del agente pigmentante de chicha morada, el estudio revela que el resina acrílica presentó mayor estabilidad de color a comparación de la resina bisacrílica y PMMA de autocurado. Si bien no hay muchos estudios con dicho agente pigmentante Santillán Tello V. (2015) ${ }^{(20)}$ compara 2 diferentes resinas mencionando que la chicha morada presenta una menor pigmentación que el café controlándolo en un periodo de 7 días.

Acuña $E$ et al. (2016) (26) también ve la estabilidad de color de las resinas, posterior a un aclaramiento dental, compara chicha morada, té verde y agua destilada, teniendo como resultado que la chicha morada causó mayor cambio de color en las muestras, pasado los 7 días el mismo resultado y a los 28 días no se encontró diferencia con los demás agentes ni grupos.

Así mismo, Trejo Jacho P. (2017) ${ }^{(27)}$ compara el grado de pigmentación en resinas nanohíbridas con y sin pulido, con café, té negro y chicha morada, siendo esta última la que tiene menor efecto de pigmentación. Peguero W. (2016) ${ }^{(28)}$ evaluó el cambio de color de una resina bisacrílica con agentes pigmentantes como el café, agua destilada, vino tinto y refresco rojo. Llegando a la conclusión que todos los agentes tuvieron significancia con el cambio de color, desde la toma inicial, teniendo el mayor grado de tinción a los 7 días y que el agente que ocasionó mayor cambio fue el café.

Christiani J. et al. (2015) ${ }^{(16)}$ coincide con la investigación actual, concluyendo que la resina bisacrílica es menos estable que la resina acrílica. Si bien es cierto la resina bisacrílica en los primeros días tiene mayor estabilidad, pasado estos y conforme al agente pigmentante expuesto, llega a ser la más inestable.

Según los resultados encontrados en este estudio, se llegó a las siguientes conclusiones:
La resina acrílica Alike tuvo mayor estabilidad de color a los 7 días al ser sumergida en agua destilada seguida por la resina bisacrílica Luxatemp Star y el PMMA de autocurado Vitalloy.

Sin embargo, a los 28 días, fue la resina bisacrílica Luxatemp Star, la que tuvo mayor estabilidad seguida por la resina acrílica Alike y PMMA de autocurado Vitalloy.

La resina acrílica Alike presentó mayor estabilidad de color en comparación con el PMMA de autocurado Vitalloy y la resina bisacrilica Luxatemp Star, al ser sumergidas en los agentes pigmentantes café y chicha morada, tanto a los 7 como a los 28 días.

El café causó la menor estabilidad de color por su pigmentación en los tres materiales dentales provisorios, independientemente de los intervalos de tiempo de almacenamiento de las muestras. El intervalo de tiempo de almacenamiento se mostró directamente proporcional al cambio de color.

Según los resultados observados en este estudio, se recomienda que si se ha planificado la utilización de algún material dental provisorio por más de un mes, tomar las precauciones respectivas sobre su cambio de color.

Así mismo, es importante seguir de manera minuciosa el protocolo recomendado por el fabricante para el manejo de los materiales dentales provisorios en la confección de las diferentes restauraciones provisionales. Al momento de realizar el estudio, se recomienda sellar herméticamente las muestras para evitar alguna contaminación externa.

Contribuciones de auditoría: (SYCHB) diseñó el estudio, realizó la revisión de literatura con el apoyo de (KAR) brindando asesoría técnica; (SYCHB) hizo el aporte del material de estudio, la recolección y obtención de datos; (RMV) dio el asesoramiento en el análisis estadístico, recolectó los datos y analizó los resultados. (RMV) y (SYCHB) hicieron la revisión crítica del artículo. Todos los autores aprobaron el manuscrito.

Fuentes de Financiamiento: Los autores financiaron la investigación. 
Conflictos de interés: Los autores declararon no tener conflicto de interés en la publicación de este articulo.

\section{REFERENCIAS BIBLIOGRAFICAS}

1. Mickeviciute E, Ivanauskiene E, Noreikiene V. Invitro Color and roughness stability of different temporary restorative materials. SBDMJ. 2016; 18(2):66-72.

2. Prasad DK, Alva $\mathrm{H}$, Shetty M. Evaluation of color stability of provisional restorative materials exposed to different mouth rinses at varying time intervals: an in-vitro study. J-IPS. 2014 Mar; 14(1):85-92.

3. Blasi $\mathrm{A}$, Barrero $\mathrm{CH}$. Color stability of provisional materials used in prosthodontics: an in-vitro study. Univ Odontol. 2011 Jul-Dic; 30(65): 8-23

4. Doray PG, Wang X, Powers JM, Burgess JO. Accelerated aging affects color stability of provisional restorative materials.

Prosthodont. 1997 Sep; 6(3):183-8.

5. Sham AS, Chu FC, Chai J, Chow TW; Color stability of provisional prosthodontic material s. J Prosthet Dent. 2004 May; 91(5):447-52.

6. Doray PG, Li D, Pwers JM. Color stability of provisional restorative materials after accelerated aging. J Prosthodont. $2001 \mathrm{Dec} ;$ 10(4):212-6.

7. Mazaro J, Minani L, Zavanelli A, Mello C, Lemos C. Evaluation of color stability of different temporary restorative materials. Revista de odontologia da unesp. 2015;44(5):262-7

8. Laura M. Estudio in-vitro de la dureza superficial de resinas acrílicas usadas en provisorios [Título Profesional de Cirujano Dentista].Lima (PE): Universidad Nacional Mayor de San Marcos; 2016.

9. Pazmiño D. Estudio comparativo in vitro de la resistencia a la deflexión transversa de acrílico para base de dentadura con y sin insertos metalicos [Grado Académico De Odontologo]. Quito (EC): Universidad Central del Ecuador; 2016 Mar.

10. Pascual-Moscardó A, Camps-Alemany I. Chromatic appreciation in the clinic and the laboratory. Med Oral Patol Oral Cir Bucal. 2006 Jul 1; 11(4):E363-8.

11. Hamza TA, Johnston WM, Schricker SR. Effect of polyhedral silsesquioxane (POSS) on the flexural strength and color of interim materials. J Prosthet Dent. 2014 Aug; 112(2):228-34.

12. Gujjari AK, Bhatnagar VM, Basavaraju RM. Color stability and flexural strength of poly (methyl methacrylate) and bis-acrylic composite based provisional crown and bridge auto-polymerizing resins exposed to beverages and food dye: an in vitro study. Indian J Dent Res. 2013 Mar-Apr; 24(2):172-7.

13. Martinez S. Estudio sobre la fiabilidad de medición del espectrofotómetro dental vita easyshade compact (Vita - Zahnfabrik) [Máster
En Ciencias Odontológicas]. Universidad Complutense De Madrid; 2012 Set.

14. Martínez J., Nieto $S$, Romeo M, Cañada L. Factores que determinan la percepción del color en odontología. Revista Internacional de Prótesis Estomatológica 2004; 6(3):218-25.

15. Gómez C. Estudio in-vitro sobre la estabilidad de color de las resinas compuestas dentales [Máster En Análisis Avanzado De Datos Multivariantes]. Universidad de Salamanca; 2013 Jul.

16. Christiani J, Devecchi J, Avalos LLano K, Altamirano H, Rocha M. Estabilidad de color de resinas para prótesis provisional. Revista del Ateneo Argentino de Odontología. 2015; 53(1):29-34.

17. Rutkunas V, Sabaliauskas V. Effects of different repolishing techniques on colour change of provisional prosthetic materials. SBDMJ. 2009; 11(4):105-12.

18. Costa Í, Lima E. Effect of colorant solutions on the color stability of provisional prosthetic materials. BJOS. 2018; 17:1-8.

19. Torres Loaiza, D. C., \& Zambrano Bonilla, M. C. (2018). Estabilidad del color de materiales provisionales en prótesis fija. Estudio in vitro entre resina acrílica y bis-acrílica. Revista Conrado, 14(62):111-6.

20. Santillán Tello V. Comparación in vitro de la estabilidad de color de las resinas compuestas filtek $^{\mathrm{TM}} \mathrm{z} 350 \mathrm{xt}$ y opallis ${ }^{\circledR}$ sometidas a diferentes sustancias pigmentantes: café, té, vino y chicha morada [Cirujano Dentista]. Universidad Peruana de Ciencias Aplicadas (UPC); 2015.

21. ISO 4049 Dentistry - Polymer-based filling, restorative and luting materials: 2009-02-11

22. American National Standards Institute. Ansi/Ada Specification No. 27: Direct Filling Resins. ADA; 1993

23. ISO 3696:2004 Dentistry -Agua para uso en análisis de laboratorio. Especificaciones y método de ensayo.

24. ISO TS 11405:2003 - Dental materials - Testing of adhesion to tooth structure

25. Rutkunas V, Sabaliauskas V, Mizutani H. Effects of different food colorants and polishing techniques on color stability of provisional prosthetic materials. Dent Mater J. 2010 Mar; 29(2):167-76.

26. Acuña E, Delgado-Cotrina L, Rumiche F, Tay L. Effect of the Purple Corn Beverage "Chicha Morada" in Composite Resin during Dental Bleaching. Scientifica. 2016; 2016:1-6.

27. Trejo Jacho P. Efecto de sustancia pigmentantes sobre el color de dos resinas nanohíbridas con y sin pulido. ET VITA. 2017; 12(2):832-6.

28. Peguero W, Cepeda A. Evaluación de la estabilidad del color, resistencia a la flexión y la compresión de materiales provisionales a base de bisacrilato utilizados en prótesis fija [Maestro de Prostodoncia e Implantología Oral]. Pontificia Universidad Católica Madre y Maestra, Facultad de Ciencias de la Salud Departamento de Estomatología; 2016. 
Samantha Ysabel Chuquiano Bringas

ORCID iD: (1) http://orcid.org/0000-0002-6296-9437

sam_ysabel4@hotmail.com

Khety Karen Arroyo Rivera

ORCID iD: (1) http://orcid.org/0000-0002-1262-8044

khkare20@hotmail.com

Rafael Morales Vadillo

ORCID iD: (단 http://orcid.org/0000-0002-7845-6048

rafomoralesv@hotmail.com

Copyright $\odot$ La revista. La revista Kiru es publicada por la Facultad de Odontología de la Universidad de San Martín de Porres, en Lima, Perú. 